\title{
The Effect of Psychological Stress in the Second and Third Trimesters of Pregnancy on the Early Cognitive Development of Offspring
}

\author{
Hui-Zi Ma ${ }^{1, \text { a }}$, Gai Li ${ }^{1}$, Qi-Yang Wang ${ }^{1}$, Zheng Zhou ${ }^{2}$, Li-Ping Yang ${ }^{1, *}$, Jiang-Hui Zhu ${ }^{1}$, \\ Feng-Xia Zhai ${ }^{2}$, Xin-Min Li ${ }^{1}$, Jun-Lin Hou ${ }^{1}$, De-Rong Kong ${ }^{3}$, Jun Zheng ${ }^{1}$ \\ ${ }^{1}$ School of Chinese and Western Medicine, Henan University of Traditional Chinese Medicine, Zhengzhou \\ 450046, China; \\ ${ }^{2}$ The Obstetrics of the Fist Affiliated Hospital of Henan University of TCM, Zhengzhou 450000, China; \\ ${ }^{3}$ Eighth People’s Hospital of Zhengzhou, Zhengzhou 450063, China \\ *Corresponding author e-mail: bioylp@126.com \\ amhz0548@163.com
}

Keywords: Pregnancy; Psychological stress; Offspring; Cognitive development; The early effect.

\begin{abstract}
Objective: To explore the effect of psychological stress in the second and third trimesters of pregnancy on the early cognitive development of offspring. Methods: SCL-90 was used to assess mothers' psychology in the second or third trimester of pregnancy. Montreal Cognitive Assessment was used to finish the screening of pregnant women cognition. The growth and development of offspring were screened by 0-6 years old children's development screening table. Gesell Developmental Scale was used to evaluate 0-12 months old offspring's cognitive development. Result: Compared with the control group, the cognitive level of mothers in the stress group was insignificantly higher $(\mathrm{P}>0.05)$. The development quotient level of DST in the stress group was significantly lower than that of the control group $(\mathrm{P}<0.05)$. Compared with the control group, the adaptive behavior, language, social behavior and comprehensive development quotient of the GDS were significantly lower in the stress group $(\mathrm{P}<0.05)$. Conclusion: The psychological stress during the second or third trimester of pregnancy has negative influence on $0-12$ months old offspring's cognitive development.
\end{abstract}

\section{Introduction}

Cognition is the understanding of things and know the process, namely the acquirement, organization and application of knowledge process[1]. It encompasses processes such as perception, attention, memory, language, thinking, consciousness and emotion, it is an important process in human higher nervous activity. Cognitive impairment is one or more aspects of cognitive processes, including autism, moderate to severe mental retardation, multiple disorders, brain damage and so on[2]. Studies have reported that factors affecting cognitive development are related to the delivery mode, upbringing, the time of mother-child communication and the wake-up of night[3-6]. Based on the theory of " Development of Health and Disease ", many diseases can be traced back to the fetus, that of the fetal stage is the critical period of growth and development. The changes of intrauterine environment caused by any physiological and pathological factors during pregnancy will affect the growth and development of the fetus, and even the long-term health of the offspring[7], Therefore, the purpose of this study was to explore the effect of psychological stress reaction on cognitive development of 0-12 months old offspring during the second and third trimesters of pregnancy. At the same time, it provides a scientific basis for the causes of cognitive impairment in clinical children. 


\section{Materials}

\subsection{General Materials}

Expectant mothers and their offspring of 0-12 months old were carried out from the pregnant women in the second and third trimester of pregnancy who lived in Zhengzhou for a long time from November 2015 to February 2017 in the Department of Obstetrics and Gynecology, First Affiliated Hospital of Henan University of CM.

\subsection{Inclusive criteria}

(1) Women of childbearing age, single live fetus, gestational weeks 28 weeks; (2) Pregnancy pregnant women are in good health, without chronic or major mental illness, and without family history of mental or intellectual illness; (3) Pregnant women who voluntarily cooperated in this study without adverse drug use history or pregnancy complications.

\subsection{Informed Consent}

(1) It was approved by the ethics committee of the first affiliated hospital of henan university of traditional Chinese medicine, and the hospital approved the study in August 2015; (2)Inform the subject of the research content of the project, and promise that the content of this investigation report is only used for this study, and does not involve other interests; (3)This study follows the principle of voluntary and confidentiality, obtaining the consent of subjects and signing the informed consent.

\section{Methods}

\subsection{Scale evaluation}

\subsubsection{Evaluation of maternal psychological stress}

SCL-90 was used to test the psychological stress of pregnant women and exclude pregnant women whose symptoms appeared before pregnancy. SCL-90 consists of 90 items, including a very broad area of psychiatric symptomatology ranging from feeling, emotion, thinking, consciousness, behaviors and living habits, interpersonal relationships, diet, and sleep. 10 factors were used to reflect the psychological conditions in 10 aspects. The 10 factors were somatization, obsessive-compulsive state, interpersonal relationships, depression, anxiety, hostility, fear, paranoia, psychosis and others. The factor score of the scale $(\geq 2)$ was positive. According to the scoring criteria, SCL-90 factor score $(\geq 2)$ was stress group and factor score $(<2)$ was control group.

\subsubsection{Evaluation of maternal cognitive level}

MoCA is a tool for screening cognitive dysfunction, with a total score of 30 points, no less than 26 points for the normal population, and no more than 12 years of education, one point should be added on the basis of the total score. MoCA was used to screen the pregnant women's cognition, and the score $(\geq 26)$ was included in the study.

\subsubsection{Evaluation of offspring cognitive development levels}

Two groups of offspring aged 0-12 months were assessed with the double-blind method. DST was used to screen the growth and development of the offspring, and GDS was used to evaluate the cognitive development of the offspring. Adaptive behavior is the most important energy area, mainly used to reflect the overall development of children, but also to reflect the organizational ability of infants to stimuli, the stimulus as a whole is decomposed into components of adaptive behavior, and these components in a meaningful way into a whole. The gross motor behavior reflects the baby's posture response and the behavior of a larger range of motion. Fine motor behavior includes the behavior of hands and fingers grasping, gripping, and manipulating objects. Language behavior reflects the infant's ability to imitate and understand other people's language. Social behavior reflects 
the infant's social and cultural manifestations of the society in which he lives. Adaptive behavior, language behavior and social behavior are mainly used to evaluate infants' cognitive level.

\subsection{Statistical Analysis}

All data was entered into Excel and analyzed using SPSS 22.0 statistical software. Measurement data are expressed by mean standard deviation, the T-test of two independent samples which follows the normal distribution and satisfies the homogeneity of variance is adopted. The test level was $\alpha=0.05, \mathrm{P}<0.05$ indicates that there is statistical difference.

\section{Results}

\subsection{Comparison of cognitive levels between two groups of pregnant women}

A total of 160 subjects were obtained. Pregnant women with SCL-90 factor $<2$ were in the control group, pregnant women with factor $\geq 2$ were in the stress group, and 80 in the control group and the stress group. Compared with the control group, MoCA scores of pregnant women in stress group were lower, and there was no significant difference $(\mathrm{P}>0.05)$. ( Table 1 )

Table 1. Comparison of MoCA scores between two groups of pregnant women( $\bar{x} \pm s, n=80)$

\begin{tabular}{ccccc}
\hline Nane & Control Group & Stress Group & $F$ & $P$ \\
\hline MoCA & $27.03 \pm 0.97$ & $26.93 \pm 0.88$ & 0.284 & 0.496 \\
\hline
\end{tabular}

\subsection{Comparison of DST developmental quotients between two groups of offspring}

Compared with the control group, the DQ of the DST of the stress group was significantly lower, and the difference was statistically significant $(\mathrm{P}<0.05)$. (Table 2$)$

Table 2. Comparison of the two groups DST offspring's development ( $\overline{\mathrm{x}} \pm \mathrm{s}, \mathrm{n}=80$ )

\begin{tabular}{cc}
\hline Group & DQ \\
\hline Control Group & $80.88 \pm 10.15$ \\
Stress Group & $76.40 \pm 8.94 *$ \\
$F$ & 1.574 \\
$P$ & 0.004 \\
\hline
\end{tabular}

Note: $* P<0.05$

\subsection{Comparison of GDS energy regions between two groups of progeny}

Compared with the control group, the adaptive behavior, language behavior and social behavior of the offspring GDS in the stress group were lower, and the difference was statistically significant(P $<0.05$ ). (Table 3)

Table 3. GDS offspring were compared for each energy region ( $\bar{x} \pm s, n=80$ )

\begin{tabular}{cccccc}
\hline Group & adaptive & gross motor & Fine motor & language & social \\
\hline Control Group & $82.43 \pm 10.83$ & $88.51 \pm 4.88$ & $88.34 \pm 6.09$ & $84.18 \pm 9.73$ & $83.21 \pm 10.23$ \\
Stress Group & $74.30 \pm 8.79 *$ & $87.19 \pm 4.32$ & $86.43 \pm 6.53$ & $76.19 \pm 8.24 *$ & $74.48 \pm 7.79 *$ \\
$F$ & 1.681 & 1.099 & 0.080 & 0.677 & 1.307 \\
$P$ & $<0.001$ & 0.073 & 0.058 & $<0.001$ & $<0.001$ \\
\hline
\end{tabular}

Note: $* P<0.05$ 


\section{Discussion}

With the development of the model of bio-psycho-social medicine, people pay more and more attention to mental health problems. As a special group, pregnant women are vulnerable to adverse events and are more likely to have psychological problems. Psychological distress of pregnant women is a major public health problem, which not only has a negative impact on the health of pregnant women themselves [8], but also on the growth and development of their offspring[9].

This study was conducted to investigate the effects of psychological stress on cognitive development of 0-12 months old offspring in the second and third trimesters of pregnancy from the perspective of clinical epidemiology. The results showed that the intelligence level of the offspring in the stress group was lower than that in the control group, indicating that psychological stress in the second and third trimesters of pregnancy had a negative effect on the intellectual development of the offspring of 0-12 December age group; The adaptive behavior, speech behavior and social behavior scores of the offspring of stress group were lower than those of control group, which showed that psychological stress in the second and third trimesters of pregnancy had adverse effects on the cognitive function of adaptive ability, speech ability and social ability of children of 0-12 December age. Above all, this study shows that psychological stress in the second and third trimester of pregnancy has adverse effects on cognitive development of children aged 0-12 months. Our research group has found through animal experiments that fear stress during pregnancy can cause cognitive retardation of its offspring [10-11], which is consistent with the results of this clinical study. Similar studies had been reported in other studies, Anita Nath et al. [12]found that psychological stress during pregnancy can have a negative impact on the growth and development of infants 10 days, 3 months, 8 months and 12 months after birth. Davis et al. [13] analyzed the relationship between cognitive development of 125 full-term infants at 3, 6 and 12 months postpartum and gestation specific anxiety of pregnant mothers, and found that the increased level of gestation specific anxiety of pregnant mothers in early pregnancy was significantly correlated with the lower cognitive development score of infants at 12 months.

In conclusion, psychological stress in the second and third trimester of pregnancy has a negative effect on cognitive development of offspring. Whether the cognitive development of offspring in the second and third trimesters of pregnancy will continue into childhood or even adulthood still needs further study. In addition, the mechanism of the effect of psychological stress on cognitive development of offspring in the second and third trimester is still not clear, which needs further study and exploration.

\section{Acknowledgments}

This work was financially supported by the National Natural Science Foundation (No.81373790) Scientific and Technological Tackling Key Project of Henan Province (No.182102310263).

\section{References}

[1] JIANG Xin-ye, MA Yu-jie, CHEN Ya-fen, et al. Effect on influencing factors on cognitive development in 480 normal infants[J]. Chinese Journal of Child Health Care, 2013,21(12): 1321-1323.

[2] YU Su-hong. The Alternate Assessment Of Children With Severe Cognitive Disabilities In The U.S. [J]. Chinese Journal of Special Education,2010,4:42-46.

[3] LIU Ting-Ting, HUANG Liang. Effects of different delivery modes on infantile growth and development[J]. Maternal and Child Health Care of China, 2017, 32(15):3536-3539.

[4] Bian Qiu-xiang, Zhang Xin-hua. Analysis on the developmental screening tests of infants [J]. Modern Medicine Journal of China, 2014, 16(10):41-42. 
[5] ZHANG Li, XU Xiao-juan, ZHU Ting, et al. Effects of maternal-child communication time on cognitive development in infants and toddlers[J]. Chinese Journal of Child Health Care, 2014,22(9):918-928.

[6] WANG Ai-ping, SUN Wan-qi, LUO Xiao-ping, et al.The association between night waking and infant cognitive development [J]. Journal of Clinical Pediatrics, 2014,32(11):1064-1068.

[7] Barker DJ. The developmental origins of chronic adult disease[J]. Acta Paediatr Suppl, 2004,93(446):26-33.

[8] Cardwell MS. Stress: pregnancy considerations. Obstet Gynecol Surv. 2013,68(2):119-129.

[9] Webb R, Ayers S. Cognitive biases in processing infant emotion by women with depression, anxiety and posttraumatic stress isorder in pregnancy or after birth: A systematic review[J]. Cogn Emot, 2014,4:1-17.

[10] YANG Li-ping, YUAN Fu-ning, LI Xin-min, et al. Effect of terror stress of adult female rats on spatial learning and memory abilities in neonatal offspring[J].Chinese Journal of Public Health,2013,29(2):214-216.

[11] WAN Hai-jiao. Research of the short and long term influence of horror injuring the kidney on offspring's emotion and congnition development based on microdialysis technology [D]. Henan College of Traditional Chinese Medicine, 2016.

[12] Anita Nath, Gudlavalleti Venkata Satyanarayana Murthy, Giridhara R. Babu, et al. Effect of prenatal exposure to maternal cortisol and psychological distress on infant development in Bengaluru, southern India: a prospective cohort study[J]. BMC Psychiatry, (2017)17:255.

[13] Elysia P. Davis, Curt. Sandman. The Timing of Prenatal Exposure to Maternal Cortisol and Psychosocial Stress Is Associated With Human Infant Cognitive Development[J]. Child Development, 2010,81(1):131-148. 